\title{
A new modification of LEACH for efficient energy in WSN
}

\author{
Taous Lechani $^{1}$, Victor Tourtchine ${ }^{2}$, Said Amari $^{3}$ \\ ${ }^{1,2}$ LIMOSE Laboratory, Faculty of Sciences, University M'Hamed Bougara of Boumerdes, Algeria \\ ${ }^{3}$ ENS Ecole normale supérieure de Cachan, France
}

\begin{tabular}{l}
\hline \hline Article Info \\
\hline Article history: \\
Received Jan 30, 2020 \\
Revised May 14, 2020 \\
Accepted Jun 1, 2020
\end{tabular}

Keywords:

Clustering

Energy efficiency

LEACH protocol

Network lifetime

Wireless sensor networks

\begin{abstract}
The limited energy of nodes in wireless sensor networks and the impossibility of replacing their batteries, have lead to protocols development which optimize and balance the energy consumption over the network. LEACH is the most used hierarchical protocol. However, one major weakness of the LEACH protocol lies in both of its random cluster formation and cluster head election. In this paper, we present two new protocols based virtual grid clustering on coverage area. In the first one, sensing area is devided into grids as squares named as VSG-LEACH and in the second one into grids as hexagons named as VHG-LEACH. In each zone, one cluster head is elected according to its residual energy and its distance from the cell center. The simulation results show that the network lifetime is prolonged by $169.67 \%$ and the energy consumption is improved by $80.97 \%$ compared to LEACH protocol.
\end{abstract} All rights reserved.

\section{Corresponding Author:}

Taous Lechani,

Laboratory of Computer Science, Modeling, Optimization and Electronic Systems (LIMOSE),

Faculty of Sciences, University M'Hamed Bougara of Boumerdes, Algeria.

Email: t.lechani@univ-boumerdes.dz

\section{INTRODUCTION}

Over the past decade, the use of Wireless Sensor Networks (WSNs) has grown significantly. They have a wide range of applications such as: military, environment monitoring, agriculture, medical care, smart home, etc [1]. WSNs are composed of a large number of tiny nodes connected according to certain architecture. Technological progress offers possibilities of integrating three functions: sensing, processing and sending data to the base station in a tiny node. Autonomy and self configuration are the main advantages of WSNs. In fact, the nodes are distributed randomly and the network is formed without pre-established support. On the other hand, the weakness of WSN consists in their limited energy and the impossibility of changing their batteries. Therefore, a balanced management of their energy is required. For this purpose, several hierarchical and flat protocols have been developed in order to minimize and balance their energy consumption [2-3]. LEACH (Low Energy Adaptive Clustering Hierarchy) is the most used hierarchical routing protocols. However, LEACH has some disadvantages mainly due to the randomness of the cluster heads election. Actually, the number of clusters per round deviates greatly from the optimum value and the clusters size is very variable, in terms of of nodes number and cluster area. Moreover, $\mathrm{CHs}$ are not necessarily spread evenly throughout the network. All these imperfections lead to an unbalanced energy consumption and reduce network lifetime.

In this paper, we present two new protocols which outperform LEACH performances. They are based on the creation of virtual grid on the coverage area. The principle consists in fixing the cluster's number and consequently, fixing the cluster heads' number $(\mathrm{CH})$. Within the first protocol, VSG-LEACH (Virtual Square Grid LEACH) protocol we use the virtual squar grid. We divide the monitored space into squares of equal size and elect one cluster head inside each square. Within the second protocol, VHG- 
LEACH (Virtual Hexagonal Grid LEACH) we have partitioned the monitored area into regular hexagons and then, adjust the membership of outside hexagon nodes. One cluster head is also elected inside each hexagon. The paper organization is as follows: The literature survey is presented in Section 2, the energy dissipation radio model is presented in section 3. A detailed description of our algorithms VSG-LEACH and VHG-LEACH is given in section 4. The simulation results and their analyses are presented in Section 5. The conclusion with future developments is presented in section 6 .

\section{LITTERATURE SURVEY}

Recently several works related to clustering have been presented and many algorithms have been developed in this concern. LEACH and its variants are the most used protocols. The cluster heads are selected randomly. LEACH protocol works by round. Each round is divided into a setup phase and a steady phase. In the setup phase, the clusters are organized and the cluster heads are elected. At the beginning of each round, each node of the network generates a random number belonging to [0, 1]. If the generated number is less than the threshold T(n) given by (1), the node elects itself as cluster head, otherwise it becomes common node [4].

$$
T(n)=\left\{\begin{array}{c}
\frac{P}{1-P\left(r \operatorname{rmod}\left(\frac{1}{P}\right)\right)} \text { if } n \in G \\
0 \text { otherwise }
\end{array}\right.
$$

Where $P$ is the percentage of cluster heads, $r$ is the round number and $G$ is the set of nodes that have not been selected as $\mathrm{CHs}$ in the last 1/P rounds. Thus, the rotation of the rule of cluster head leads to balanced energy consumption; hence, it maximizes the time until the death of the first node. Each common node joins one $\mathrm{CH}$ based on the signal strength.

In the steady setup phase, data are sent to the base station. The steady phase is divided into frames. In each frame, common nodes send their sensing data to $\mathrm{CH}$ according to scheduled time slots. In order to save energy, the nodes remain in a sleep mode while waiting for their turn to send data. The cluster heads aggregate the data and relay it to the base station (BS). Knowing that data transmission over long distances is the most energy consuming network, clustering reduce energie consumption by reducing transmission distances.

In this work, we review the LEACH protocol and its extended versions from energy efficiency viewpoint. There are many works focused on improving LEACH protocol. Numbers of them propose new formulas of threshold which take into account the residual energy of the nodes and their distances from the base station, including LEACH-C, LEACH-V, LEACH-R and LEACH-M [5-8]. Several cluster heads selection algorithms based on the distance have been proposed [9-10]. LEACH-DT (Distance based Threshold) [8], rather than using a heuristic approach, proposes a distributed LEACH based $\mathrm{CH}$ selection algorithm. Nodes are self selected to be $\mathrm{CH}$ with different probabilities based on their distances from the Base Station (BS). Recently, Modified LEACH-DT is another proposed algorithm [11-12], which has improved the LEACH-DT consedering both distance and residual energy in cluster heads election. The proposed approach ensures better energy balance of the network nodes. In LEACH protocol, energy consumption depends on distances of nodes to $\mathrm{CHs}$ and base station. Thus, the cluster heads which are far from the base station deplete their energy earlier than those close to the base station. This modified protocol improves LEACH-DT by according higher probability to closer nodes to become cluster heads.

SEECP (Stable Energy Efficient Clustering Protocol for Wireless Sensor Networks) extends the stable part of the network lifetime in order to preserve its coverage [13]. This reactive protocol transmits the data to the base station, only in the case where a threshold value is exceeded. In order to minimize energy consumption, remote nodes from the base station transmit their data by multi-hop. AECR (Adaptative Energy Aware Cluster-Based Routing) protocol avoids the random formation of clusters and generates clusters of homogeneous size [14]. It eliminates the possibility of election of two close nodes as CHs and optimizes inter and intra-cluster routing. It also calculates the maximum distance from which the multi-hop is more energy efficient.

An energy-balanced clustering routing algorithm for WSN is proposed in [15]. It is an advanced multi-hop routing protocol which operates as follows: when the cluster heads are close to the base station they directly communicate with it and use multi-hop communication when they are far from it. In [16], the authors improved LEACH by selecting a secondary cluster head, if the energy of the cluster head is less than the average energy of all network nodes. In this case, the secondary cluster head is responsible for receiving and fusing the data collected from the member nodes and sending it to its cluster head which redirects it to the BS. A Leach-VM protocol based on weight and intra-cluster multi-hop mechanism is proposed in [17].

Indonesian J Elec Eng \& Comp Sci, Vol. 20, No. 3, December 2020 : 1495 - 1506 
It consists of electing a weight relay in the cluster, according to the member nodes residual energy and their distances from the base station. The cluster head sends aggregation data to its weight relay which forwards them to the BS.

In [18], authors propose a new cluster head selection algorithm for WSN which tries to stabilize the CH's number, while favoring the election of nodes with high residual energy. Before being designated as $\mathrm{CH}$, a node verifies the number of $\mathrm{CHs}$ already existing through the variable $\mathrm{CH}$ heard (according to received messages). If this variable is less than an optimal value, then the node declares itself as $\mathrm{CH}$ and sends membership requests; otherwise as a member node and looks for the best cluster to join.

Multicriteria based cluster head selection is proposed in [19]. The authors improve the WSN performance by considering five different parameters to select the most optimum node to be cluster head. These parameters are energy level, distance from neighboring nodes, distance from center of the cluste, number of times a node has been zone head and whether a node is merged. The whole network is divided into virtual grids where each grid represents a cluster then cluster will be merged or splitted depending on its density.

In [20], the network is subdivided into various regions based on the distance of BS from each sensor node. In some regions, LEACH protocol is implemented and in the others, nodes transmit directly their data to a rechargeable Sub-BS placed inside the network or the the BS placed outside the network. The same protocol based on sub-netting technique is applied for Heterogeneous network and network lifetime is analyzed considering four different position of Sub-BS [21].

The clustering transforms the global communication into a local communication in order to reduce the distances of transmissions, and thus minimizes the energy consumption. A judicious choice of the number of $\mathrm{CHs}$ is required. Increasing the number of clusters leads to an increased aggregation energy. On the other hand, reducing the number of clusters increases the number of member nodes per cluster and consequently, increases the energy of communication because of the great distances and the energy of aggregation due to the load on the CHs. Hence an optimum number of clusters is required to ensure best performance in the network. Energy efficiency and network lifetime are the most important metrics in evaluating network performances. Lifetime of WSN is most commonly defined as the time elapsed until the first node in the network depletes its energy. There are other definitions for $25 \%, 50 \%$ or $75 \%$ of alive nodes.

\section{RADIO ENERGY MODEL}

The energy consumption model used in this work is based on first order model introduced by Heinzelman [4]. The transmitter dissipates energy to transmit and amplify the signals. The receiver dissipates energy only to receive data. Figure 1 shows the energy model consumption.

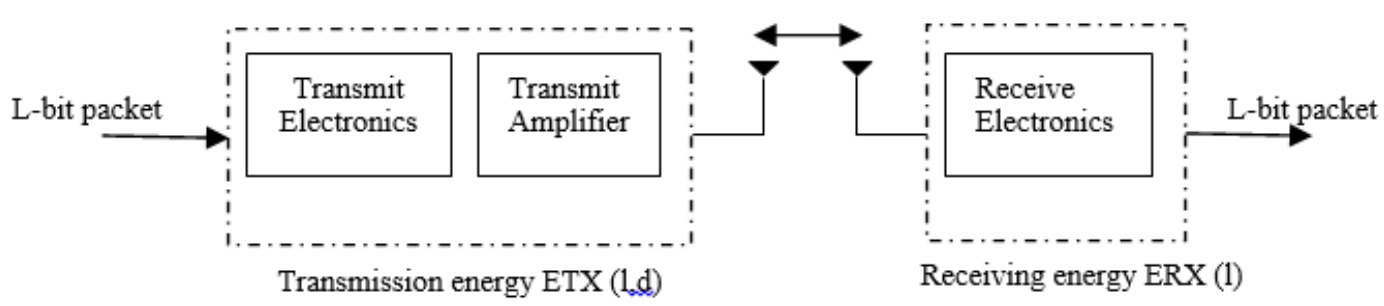

Figure 1. Radio energy dissipation model

$$
\begin{aligned}
& E_{T X}(l, d)=\left\{\begin{array}{c}
l * E_{\text {elec }}+l * \varepsilon_{f s} * d^{2} \text { if } d<d_{0} \\
l * E_{\text {elec }}+l * \varepsilon_{\text {two-ray }} * d^{4} \text { if } d \geq d_{0}
\end{array}\right. \\
& E_{R X}=l * E_{\text {elec }} \\
& d_{0}=\sqrt{\frac{\varepsilon_{f s}}{\varepsilon_{t w o-r a y}}}
\end{aligned}
$$

- ETX: energy spent to transmit 1 bits;

- ERX: energy spent to receive 1 bits;

- Eelec: energy spent to transmit or receive one bit; 
- $\varepsilon f s:$ amplification factor for free space model;

- etwo-ray: amplification factor for two-ray model;

- d: distance between transmitter and receiver;

- d0: crossover distance;

- 1: number of data bits.

\section{NEW PROPOSED PROTOCOLS}

In this section, we explain our approach and detail the two proposed protocols.

\subsection{Tessellation}

We believe the ideal form to partition a plane into regular shapes is the circle. However, it does not allow paving a surface without leaving holes and without overlapping each other. In this work, we seek to partition a plane into regular shapes which can be arranged according to two conditions:

1. The shapes should fit together without any gaps.

2. The shapes should not overlap.

Lemme: There are only three regular tessellations of the plane and the honeycomb is the optimal shape for the space occupation.

Proof: Consider a regular polygon with $\mathrm{n}$ sides and $\theta$ is the angle between two consecutive sides. The polygon is divided into $\mathrm{n}$ identical isosceles triangles as shown in Figure 2.

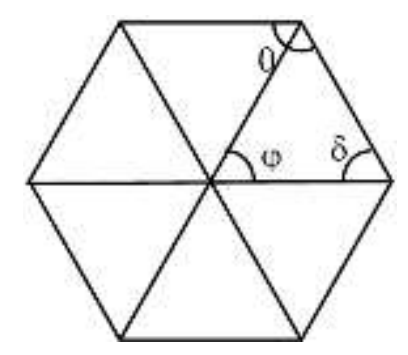

Figure 2. Regular polygon.

We have $\delta+\varphi+\theta / 2=\pi$ where $\varphi=2 \pi / \mathrm{n}$ and $\delta=\theta / 2$

Thus $\theta+\frac{2 \pi}{\mathrm{n}}=\pi$

$\theta=\frac{(\mathrm{n}-2) \pi}{\mathrm{n}}=\frac{2 \pi}{\mathrm{m}}$ With $\mathrm{m}$ natural integer

Thus $(n-2)(m-2)=4$

So (n-2) must be a divisor of 4 , thus $n$ can be equal to 3 or 4 or 6 .

Therefore equilateral triangles, squares and regular hexagons are the only regular convex polygons that can pave the plane. Intuitively, we choose to study square and hexagonal shapes.

\subsection{Proposed Protocols}

In this section, we give a detailed description of the new virtual square grid LEACH (VSG-LEACH) and virtual hexagon grid LEACH (VHG-LEACH) protocols. The main idea is to overcome the randomness of cluster formation and cluster heads election in LEACH protocol [19], [22]. The virtual grid of the sensing area ensures an even distribution of cluster heads over the whole network and fixing the cluster head number at its optimum value. This approach distributes uniformly the work-load on part of cluster head nodes which therefore extends network lifetime. The $\mathrm{CH}$ optimal number is in the same proportion as that proposed in LEACH which considers 5\% of the total number of sensor nodes. These proposed algorithms have been developed on the basis of static cluster and dynamic cluster head. Compared to LEACH, the choice of static clusters ensures the saving of the energy consumed during the clusters forming and fixing the number of clusters at its optimal value $\mathrm{z}_{\mathrm{opt}}$ given by [4]: 


$$
\mathrm{z}_{\text {opt }}=\sqrt{\frac{\mathrm{N}}{2 \pi}} \sqrt{\frac{\varepsilon_{\mathrm{fs}}}{\varepsilon_{\text {two-ray }}}} \frac{\mathrm{M}}{\mathrm{d}_{\text {to BS }}^{2}}
$$

For a WSN with $\mathrm{N}$ nodes, the sensor field is partitioned into $\mathrm{Z}$ equal sized zones [23-25] where $\mathrm{Z}$ is a square number given by (6). Figure 3 (a), (b) and (c) shows network partitioning into various uniform sized cells for $\mathrm{N}=100,200$ and 300 respectively.

$$
Z=\left\{\begin{array}{c}
4 N \times 0.05 \leq 6 \\
96<N \times 0.05 \leq 12 \\
1612<N \times 0.05 \leq 20 \\
\vdots \\
\vdots
\end{array}\right.
$$

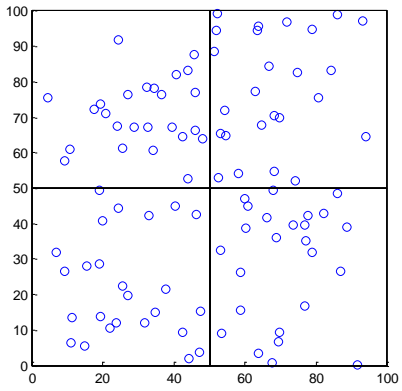

(a) $\mathrm{Z}=4$ while $\mathrm{N} \leq 100$

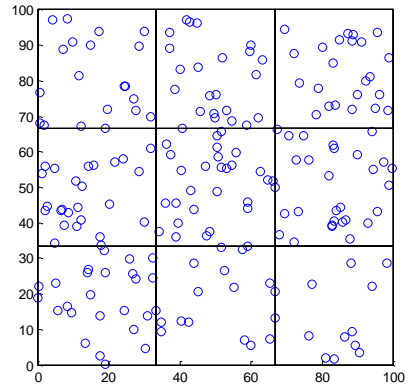

(b) $\mathrm{Z}=9$ while $101 \leq \mathrm{N} \leq 200$

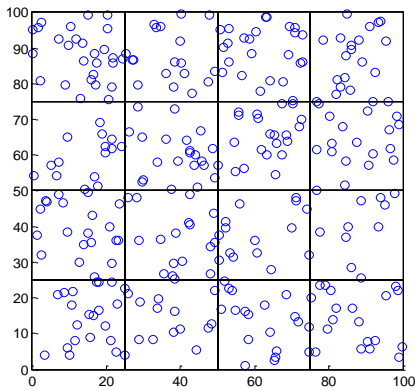

(c) $\mathrm{Z}=9$ while $201 \leq \mathrm{N} \leq 300$

Figure 3. Network partitioning into various uniform sized cells

Contrary to VSG-LEACH, the nodes can be outside the regular hexagons. In this case, we adjust membership of outside nodes as to balance clusters. In order to reduce the energy consumption, we also permit to alone nodes for which the distance to the base station is lower than the distance to the nearest cluster center, to send directly their data to the sink. The communication in these protocols uses the round as a basic unit. Each round is made up of setup phase and steady state phase which is longer than the setup one. The time for each round is $t_{\text {round }}[4]$ and is defined by (7).

$$
\mathrm{t}_{\text {round }}=10 . \mathrm{E} 0
$$

Figure 4 shows the constitution of one round of these protocols.

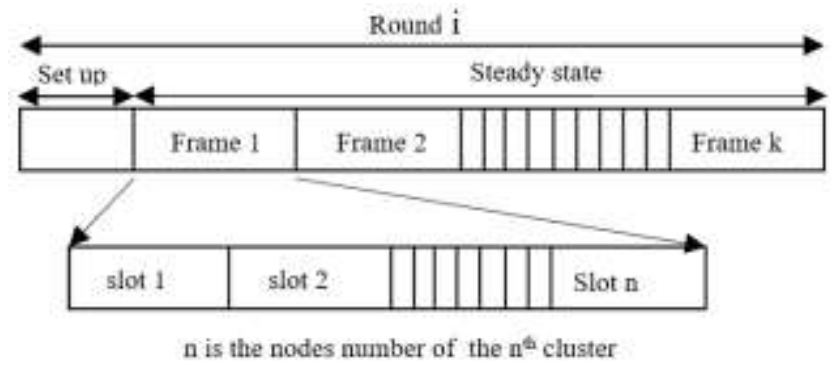

Figure 4. Round in VSG-LEACH and VHG-LEACH

\subsubsection{Setup phase}

During this phase, a cluster head of each cell (square or hexagon) is designed and a schedule TDMA (Time Division Multiple Access) is created. It also assigns to each member node a slot for data transmission. At the first round, because of the same initial energy of each node, the nearest node to the center of each zone is designed as $\mathrm{CH}$. Starting from the second round, residuals energies and distances to the center of 
each zone are considered to choose the new $\mathrm{CHs}$. So, the probability of becoming $\mathrm{CH}$ is directly proportional to residual energy and inversely proportional to distance. The weight W(i) of each node (i) is calculated by the following (8).

$$
\mathrm{W}(\mathrm{i})=\alpha \cdot \text { Energy }_{\text {res }}(\mathrm{i})+\frac{\beta}{\operatorname{Dist}(\mathrm{i})}
$$

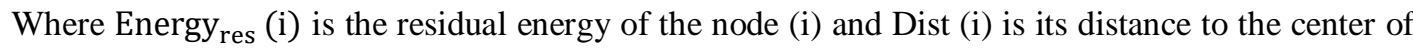
the cell. $\alpha, \beta$ are weights assigned to residual Energy and Distance to cell center according to its importance. In each cell, the node having the highest weight is selected to be the new cluster head for the next round. With this approach, nodes near to the center of the squares with high residual energy are favored to become cluster heads. Consequently, we balance the energy consumption and therefore extend the network lifetime.

\subsubsection{Steady state phase}

This phase is divided into frames. During each frame, each node sends one message to the $\mathrm{CH}$ during its slot using of the TDMA schedule. Outside their slots, their communication interfaces are switched off.

In this way, nodes save their energy. The slot time where a node transmits data is constant; so the time to send a frame of data depends on the number of nodes in the cluster. The cluster heads, from their part, receive and aggregate data then transmit them to the base station.

\subsubsection{VSG-LEACH algorithm}

The pseudo code of the VSG-LEACH algorithm is given in Algorithm 1.

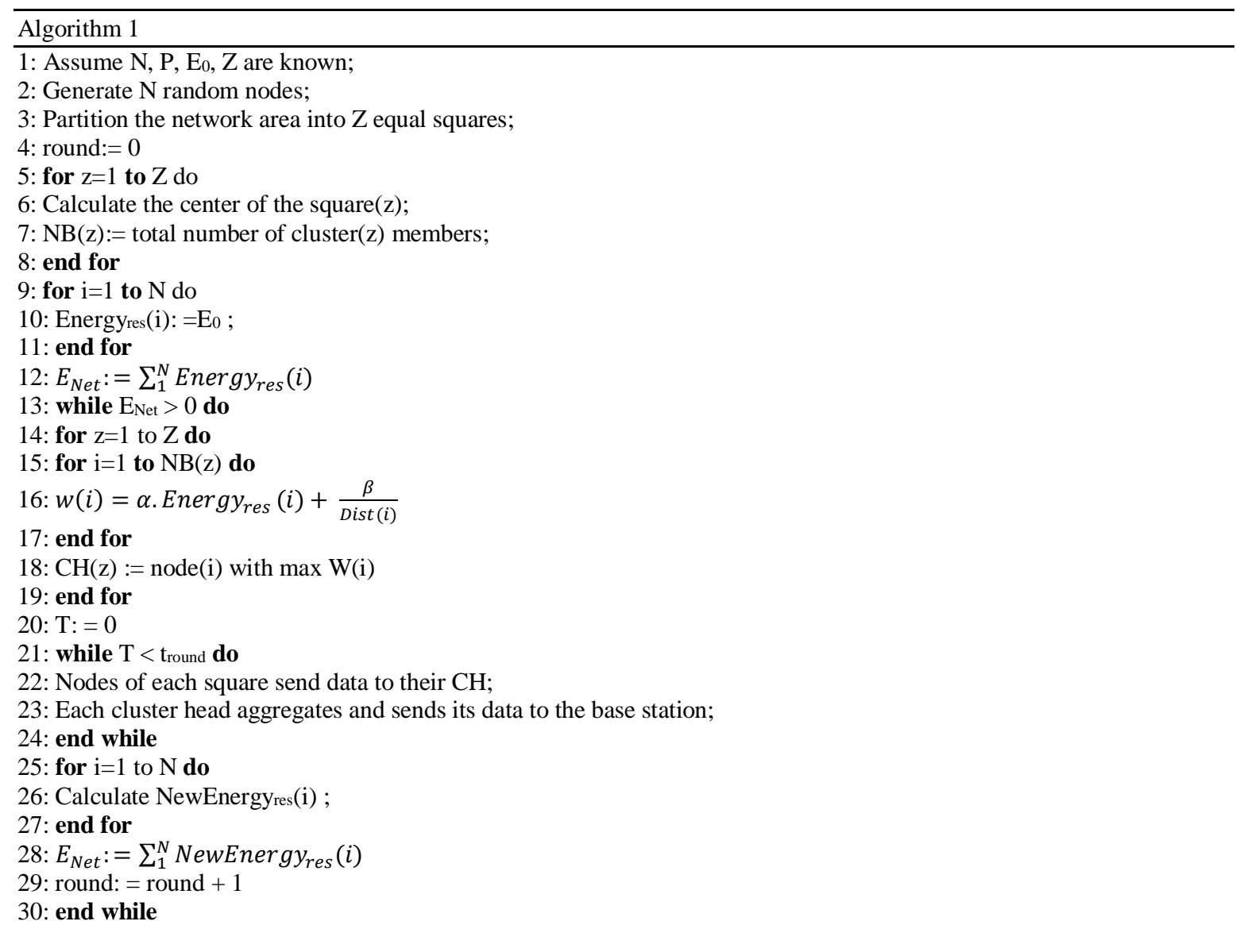

\subsubsection{VGH-LEACH algorithm}

Algorithm 2 gives the working procedure of the VHG-LEACH protocol. 


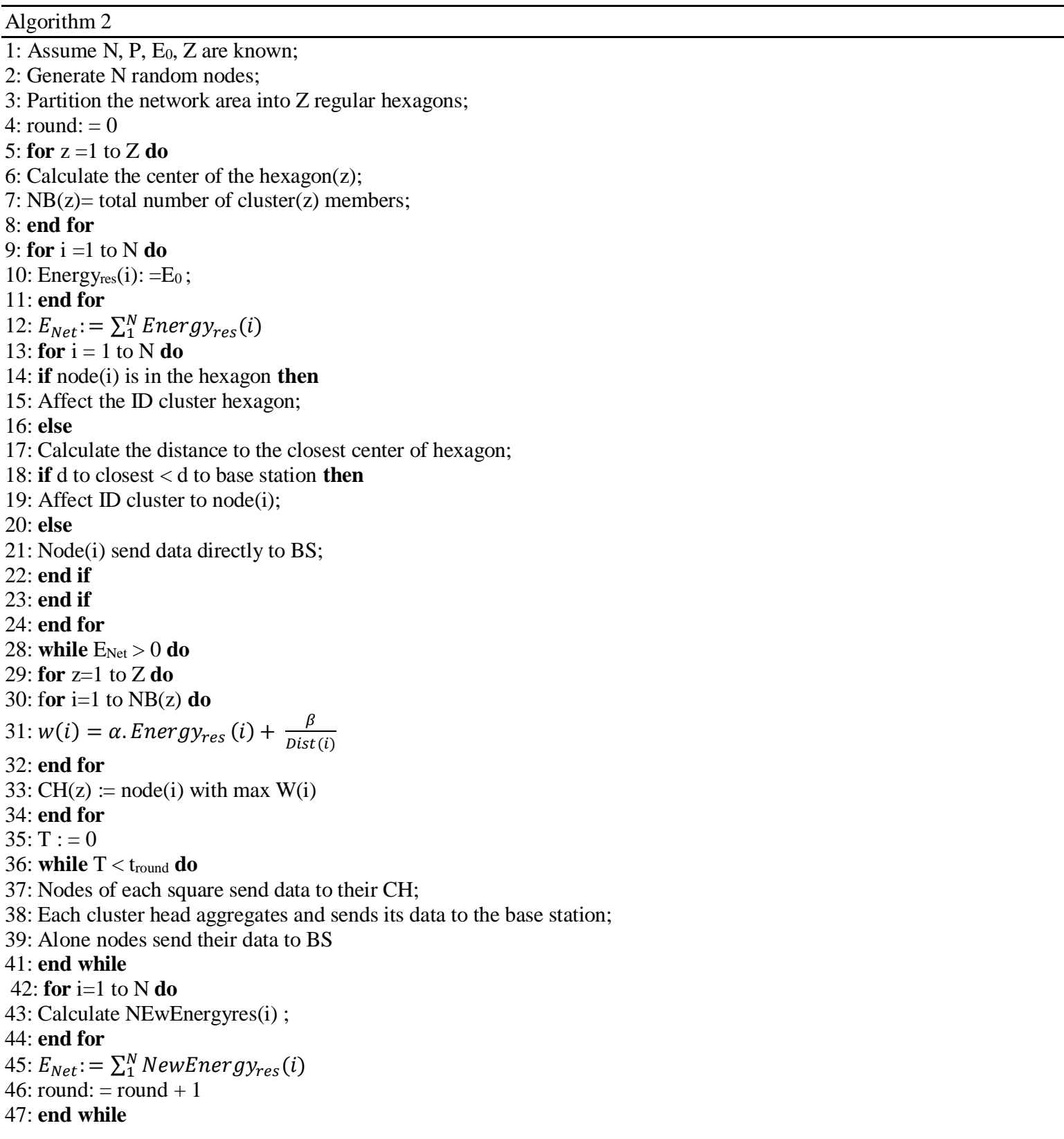

\section{SIMULATIONS AND RESULT ANALYSIS}

\subsection{Assumption and Parameters}

This paper uses Matlab R2014a as a simulation platform for LEACH protocol and for the two improved protocols (VSG-LEACH and VHG-LEACH). Initially, 100 nodes are deployed uniformly in an M x M square area. The simulations are perfomed for two base station positions which are: outside the sensing field at coordinates $(50,175)$ and the center of the sensing field at coordinates $(50,50)$.

The following assumptions are made for these simulations:

- The base station is located at fixed position and its location is known by each node.

- The location of sensor nodes are known and fixed after deployment.

- All the sensor nodes in the network are given the same amount of energy and cannot be recharged.

Used parameters are given in Table 1. 
Table 1. Simulation parameters

\begin{tabular}{ccc}
\hline Parameter & Description & Value \\
\hline $\mathrm{N}$ & Number of nodes & 100 \\
$\mathrm{M} \mathrm{x} \mathrm{M}$ & Network size & $100 \times 100\left(\mathrm{~m}^{2}\right)$ \\
$\left(\mathrm{X}_{0}, \mathrm{Y}_{0}\right)$ & Location of the base station & $(50,175)$ \\
$\mathrm{E}_{0}$ & Initial energy of nodes & $2 \mathrm{~J}$ \\
$\mathrm{P}$ & Percentage of cluster heads & $5 \%$ \\
$\mathrm{E}_{\mathrm{TX}}$ & Energy to transmit one bit & $0.5 \mathrm{~nJ} / \mathrm{bit}$ \\
$\mathrm{E}_{\mathrm{RX}}$ & Energy to receive one bit & $0.5 \mathrm{~nJ} / \mathrm{bit}$ \\
$\mathrm{EAG}$ & Aggregation energy & $5 \mathrm{~nJ}$ \\
$\varepsilon_{\mathrm{amp}}$ & Amplification factor for free space model & $10 \mathrm{pJ} / \mathrm{bit} / \mathrm{m}^{2}$ \\
$\varepsilon_{\text {two_ray }}$ & Amplification factor for free multi-path model & $0.013 \mathrm{pJ} / \mathrm{bit} / \mathrm{m}^{4}$ \\
$\mathrm{~d}_{0}$ & Crossover distance & $87 \mathrm{~m}$ \\
$\mathrm{~K}$ & Packet size & $500 \mathrm{bytes}$ \\
$\alpha, \beta$ & Weighting coefficients & 0.5 \\
\hline
\end{tabular}

\subsection{Discussions and Results Interpretation}

The deployment of 100 sensor nodes over an area of $100 \mathrm{X} 100 \mathrm{~m}^{2}$ and their clustering are shown in the three protocols. Figure 5 gives an example of clustering in LEACH round, which shows that the number of clusters deviates strongly from the optimal value five ( 9 clusters in this round). All the nodes marked with the same color belong to the same cluster. Blue squares design cluster heads and red stars represent dead nodes. We see that the nodes furthest away from the base station exhaust their energy very quickly. For a network of 100 nodes, in VSG-LEACH, the network is devided into four equal size squares as showen in Figure 6. The nodes of each square form a cluster. The second proposed protocol, VHG-LEACH, improves the WSN performances by paving the sensing area with five equal size hexagons. In Figure 7, we see the hexagonal clustering as defined in VHG-LEACH algorithm. The red crosses represent the cluster centers (square or hexagon). Figure 8 shows the variation of the number of clusters according to the round. It is clear that the number of clusters deviates strongly from the optimum value and can reach it more than 3 times. This explains the load imbalance in LEACH protocol. The simulation results are compared with the simulation results of LEACH protocol. The performance criteria used in this paper are the network lifetime and energie consumption.

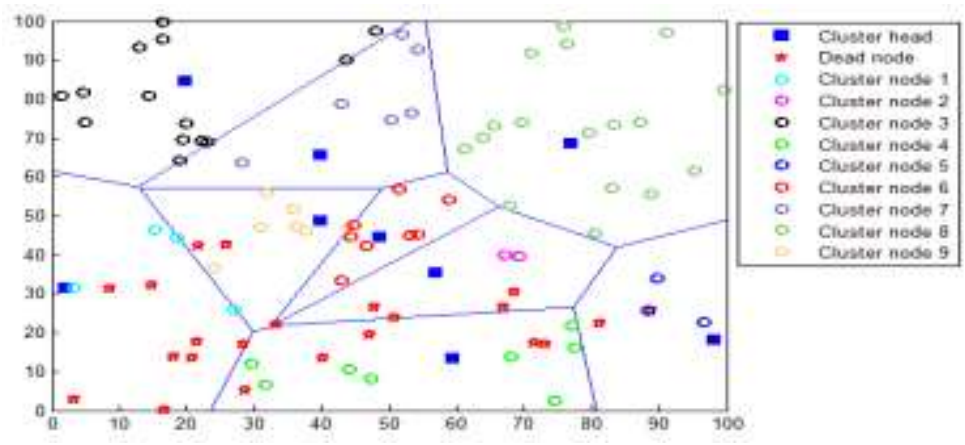

Figure 5. Example of clustering with LEACH protocol

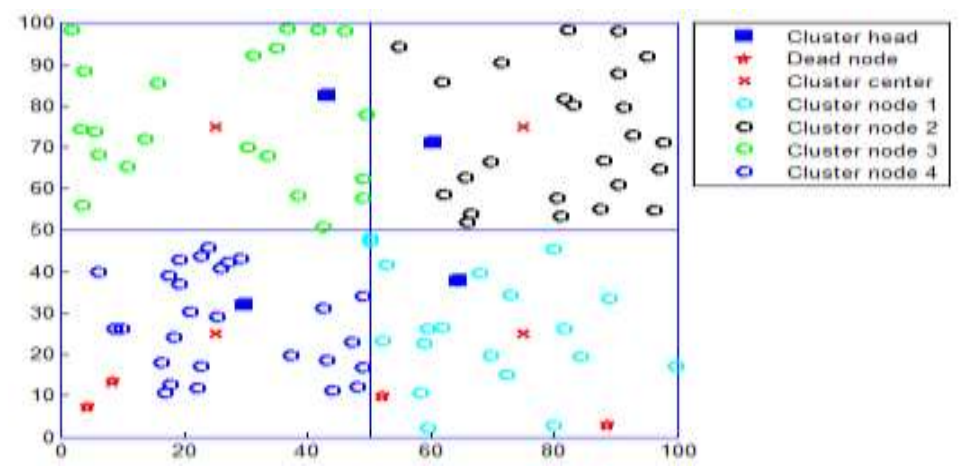

Figure 6. Clustering with VSG-LEACH protocol 


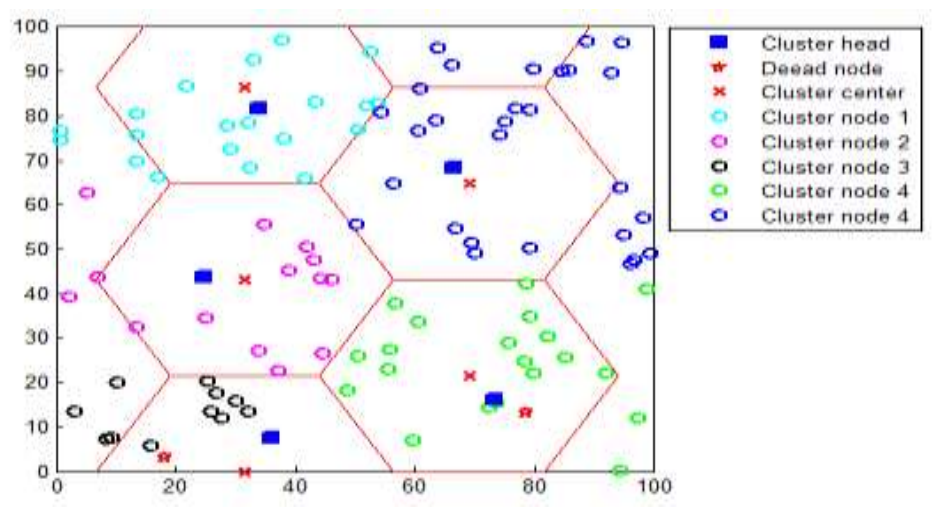

Figure 7. Example of clustering with VHG-LEACH protocol

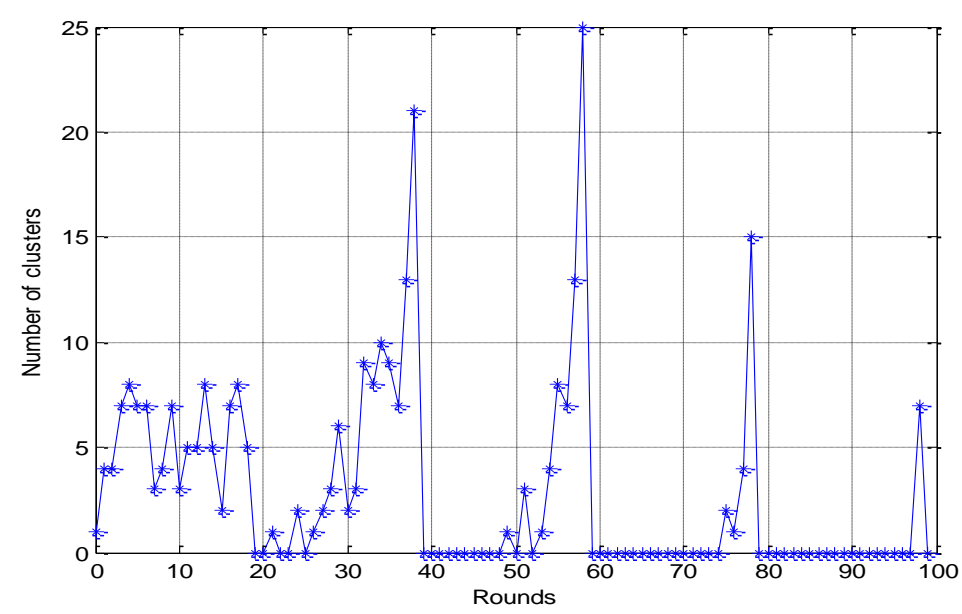

Figure 8. Number of cluster vs. round number in LEACH

\subsubsection{Network lifetime}

We define it as the elapsed time from the start to the First Node Death (FND). This parameter measures the stability period. Depending on the applications, there may be requirements on this stability period. We also measured and compared the Half Node Death (HND) and the Last Node Death (LND) which corresponds to time death of $50 \%$ nodes and all the nodes, respectively.

The network lifetime of LEACH and our two improved protocols are illustrated in Figure 9 (a) for the base station located at coordinate $(50,175)$. We observe that VSG-LEACH improves the network lifetime by $49.77 \%$ as compared to LEACH. With VHG-LEACH, FND is improved by $52.01 \%$ compared to LEACH. While the base station is located at the center of sensing area, in Figure 9 (b), we can see that VHG-LEACH extends the network lifetime by $169.67 \%$. Unlike LEACH, our results show that dead nodes are more evenly distributed in the network with VSG-LEACH and VHG-LEACH protocols. The proposed protocols improve both stable and instable periods. This is mainly due to the control of the number of clusters and the even distribution of cluster heads across the network area. Moreover, the choice of the cluster head is made inside the cluster, which saves the energy consumed during the clusters formation in LEACH protocol. Table 2 shows the network lifetime in terms of number of rounds until FND, HND and LND.

Table 2. Comparison of network lifetime of protocols

\begin{tabular}{ccccccc}
\hline BS position & \% Dead nodes & LEACH & VSG-LEACH & Rate VSG-LEACH & VHG-LEACH & Rate VHG-LEACH \\
\hline (50,175) & 1 (FND) & 671 & 1005 & $49.77 \%$ & 1020 & $52.01 \%$ \\
& 50 (HND) & 1683 & 1714 & $1.84 \%$ & 3390 & $101.42 \%$ \\
& 100 (LND) & 2680 & 2926 & $9.17 \%$ & 4850 & $80.97 \%$ \\
$(50,50)$ & 1(FND) & 1576 & 3944 & $150.25 \%$ & 4250 & $169.67 \%$ \\
& 50 (HND) & 3890 & 4474 & $15.01 \%$ & 5391 & $38.59 \%$ \\
& $100(\mathrm{LND})$ & 5802 & 5518 & $-4.89 \%$ & 6060 & $4.45 \%$ \\
\hline
\end{tabular}




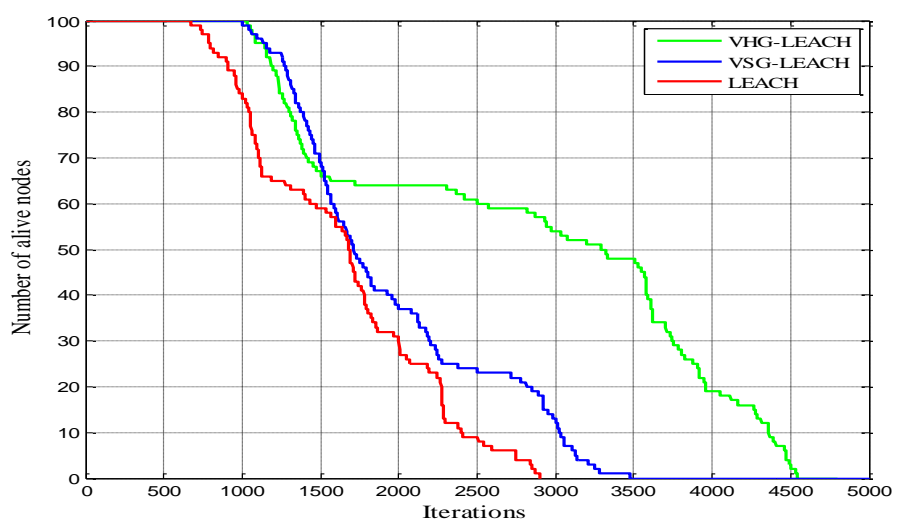

(a)

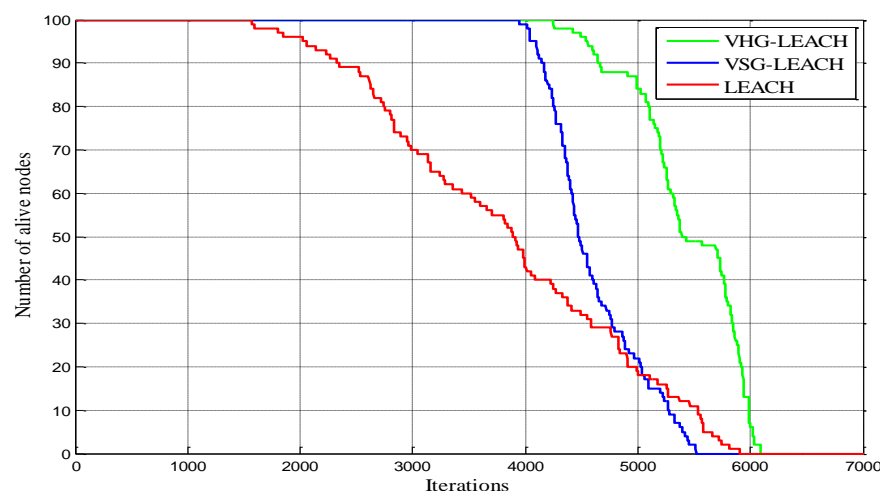

(b)

Figure 9. Alive nodes vs. iterations: a) BS $(50,175)$, b) BS $(50,50)$

\subsubsection{Residual Energy}

Figure 10 (a) shows a comparison between the total residual energy of the network while the base station is located outside the field. It is obvious that VHG- LEACH protocol ensures a higher total residual energy which means that the network could survive for a longer period of time. In our approach, the nodes with more remaining energy were selected as the $\mathrm{CH}$ more frequently than the nodes with less remaining energy, so the energy consumption was balanced. An improvement of $9.18 \%$ and $80.97 \%$ against $\mathrm{LEACH}$ is observed with VSG-LEACH and VHG-LEACH respectively. In the case where the base station is at the sensing area center, simulation results are presented in Figure 10 (b). We can see that VHG-LEACH improves residual energy consumption by $4.45 \%$. The effectiveness of VHG-LEACH is more important whith long distances. Table 3 shows the network energy consumption in terms of the number of rounds.

Table 3. Comparison of network energy consumption of protocols

\begin{tabular}{|c|c|c|c|c|c|c|}
\hline BS position & $\%$ Energy consumption & LEACH & VSG-LEACH & Rate VSG-LEACH & VHG-LEACH & Rate VHG-LEACH \\
\hline \multirow[t]{4}{*}{$(50,175)$} & 25 & 339 & 434 & $28.02 \%$ & 360 & $6.19 \%$ \\
\hline & 50 & 783 & 905 & $15.58 \%$ & 944 & $20.56 \%$ \\
\hline & 75 & 1237 & 1380 & $11.56 \%$ & 1890 & $52.79 \%$ \\
\hline & 100 & 2680 & 2926 & $9.18 \%$ & 4850 & $80.97 \%$ \\
\hline \multirow[t]{4}{*}{$(50,50)$} & 25 & 896 & 1123 & $25.33 \%$ & 1332 & $48.66 \%$ \\
\hline & 50 & 1783 & 2243 & $25.80 \%$ & 2661 & $49.24 \%$ \\
\hline & 75 & 2785 & 3363 & $20.75 \%$ & 3889 & $39.64 \%$ \\
\hline & 100 & 5802 & 5434 & $-6.34 \%$ & 6060 & $4.45 \%$ \\
\hline
\end{tabular}




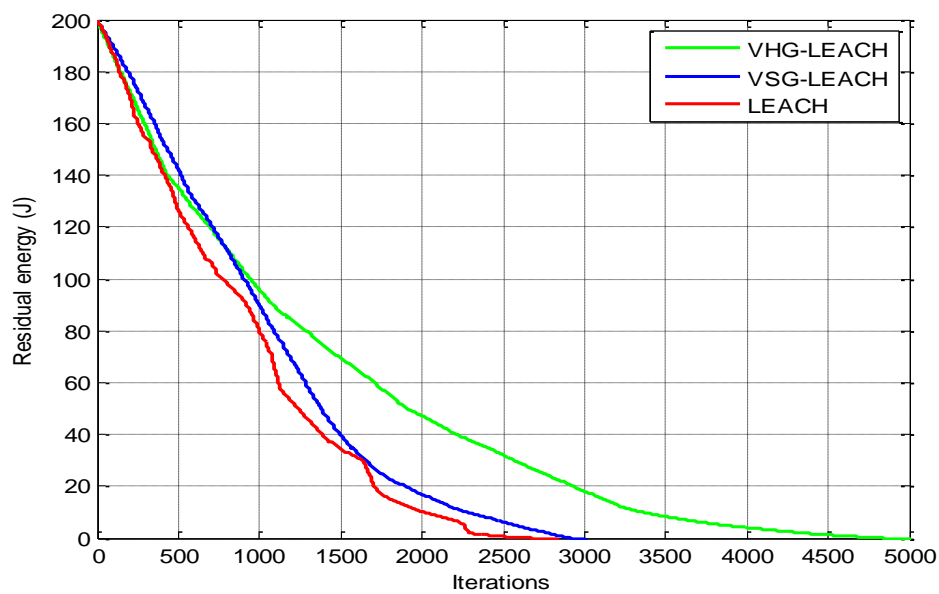

(a)

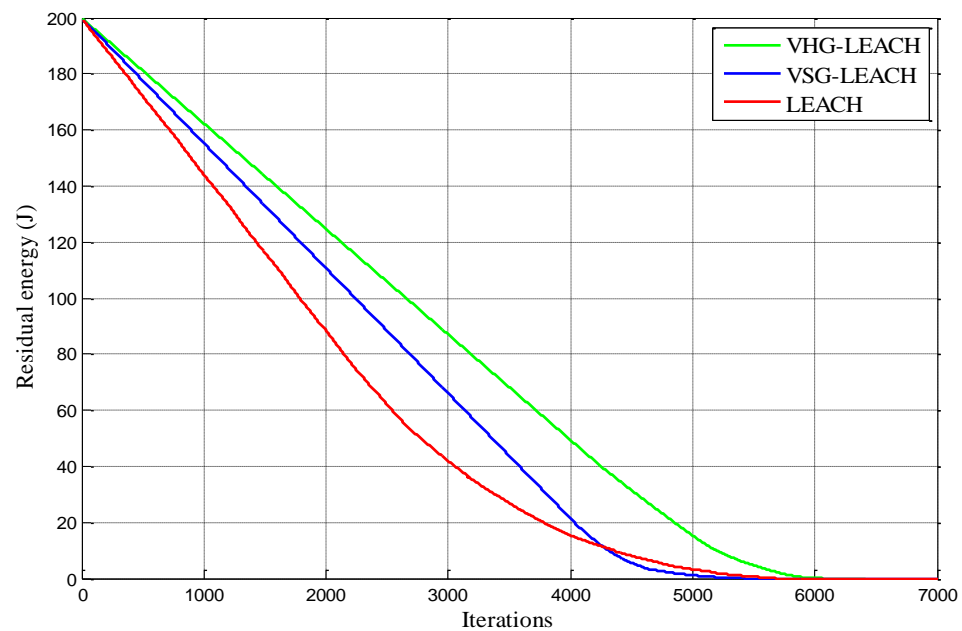

(b)

Figure 10. Residual energy vs. iterations: a) BS $(50,175)$, b) BS $(50,50)$

\section{CONCLUSION}

In this paper, two new protocols are presented in order to improve WSN performance. The two algorithms have been developed on the basis of static clusters and dynamic cluster heads. Our VSG-LEACH and VHG-LEACH partition the sensor field into a virtual grid for an even distribution of cluster heads. Fixing the clusters and varying cluster head in each cluster by taking into account residual energy and distance to BS, ensure good load balance over all the network nodes. The simulation results show that VSGLEACH and VHG-LEACH extend the network lifetime, compared to LEACH. The two proposed protocols prolong also instable period and minimize energy consumption. It is expected, with respect to current established results, that the proposed protocols give better results in large scale networks. As future work, we will study the effectiveness of this approach on heterogeneous networks.

\section{REFERENCES}

[1] I. F. Akyildiz, et al., "Wireless sensor networks: asurvey," Computer networks, vol. 38, pp. 393-422, 2002.

[2] M. M. Afsar and M. H. Tayarani-N, "Clustering in sensor networks: A literature survey," Journal of Network and Computer Applications, vol. 46, pp. 198-226, 2014.

[3] L. Kong, et al., "ICP: Instantaneousclustering protocol for wireless sensor networks," Computer networks, vol. 101, pp. 144-157, 2016.

[4] W. B. Heinzelman, "Application-specific protocol architectures for wireless networks," PhD thesis, Massachusetts Institute of Technology, USA, June 2000.

[5] M.Tripathi, et al., "Energy efficient LEACH-C protocol for Wireless Sensor Network," in Third International Conference on Computational Intelligence and Information Technology, 18-19 Oct. 2013. Mumbai, India 
[6] A. Ahlawat and V. Malik, "An extended vice-cluster selection approach to improve V LEACH protocol in WSN," in Advanced Computing and Communication Technologies (ACCT) 2013, Third International Conference on. IEEE, 2013.

[7] V. Mhatre and C. Rosenberg, "Homogeneous vs heterogeneous clustered sensor networks: a comparative study,", Communications, International Conference, 6, 2004 IEEE,

[8] J.Y. Lee and D. Lee, "Improvement of CH election in three-level heterogeneous WSN," Indonesian Journal of Electrical Engineering and Computer Science, Vol. 13 No. 1, pp. 272-278, Jan 2019.

[9] A. El Aalaoui and A. Hajraoui, "Energy efficiency of organized cluster election method in wireless sensor networks," Indonesian Journal of Electrical Engineering and Computer Science, Vol. 18 No. 1, pp. 218-226, Apr 2020.

[10] Sang H. Kang and Thinh Nguyen, "Distance Based Thresholds for Cluster Head Selection in Wireless Sensor Networks," IEEE Communications Letters, Vol. 16 No. 9, pp. 1396 - 1399, Sep 2012.

[11] V. Gupta and R. Pandey, "Modified LEACH-DT Algorithm with. Hierarchical Extension for Wireless Sensor, " I. J. Computer Network and Information Security, Vol. 8 No .2, pp.32-40, 2016.

[12] T. S. Singh, et al., "Distance Based Multi Single Hop Low Energy Adaptive Clustering Hierarchy (MS LEACH) Routing Protocol in Wireless Sensor Network, " IEEE 6th International Conference on Advanced Computing (IACC), 27-28 Feb. 2016, Bhimavaram, India.

[13] N. Mittal, et al., "A stable energy efficient clustering protocol for wireless sensor networks," Wireless Netw, Vol. 23 No. 6, pp 1809-1821, 2017.

[14] K. Haseeb, et al., "Adaptive energy aware cluster-based routing protocol for wireless sensor networks, "Wireless Netw, Vol. 23 No. 6, pp 1953-1966, 2016.

[15] F. Shang and Y. Lei, "An Energy-Balanced Clustering Routing Algorithm for Wireless Sensor Network," Wireless Sensor Network, Vol. 2 No. 10, pp. 777-783, 2010.

[16] C. FU, et al., "An Energy Balanced Algorithm of LEACH Protocol in WSN," IJCSI International Journal of Computer Science, Vol. 10 No. 1, pp.354-359, 2013.

[17] J. Zhang, et al., "LEACH-VM: weighted and intra-cluster multi-hop energy efficient algorithm for wireless sensor networks," Proceeding of the 35th Chinese Control Conference, July 27-29, 2016 Chengdu, China.

[18] P.K Batra and K. Kant, "LEACH-MAC: a new cluster head selection algorithm for Wireless Sensor Networks," Wireless Netw, Vol. 22 No.1, pp 49-60, 2016.

[19] H. Farman et al., "Multi-criteria based zone head selection in the Internet of Things based wireless sensor networks," Futur. Gener. Comput. Syst., 2018.

[20] T. M. Behera et al. "SNRP: sub-netting based routing protocol for improved network performance in WSN," International Journal of Advanced Science and Technology, Vol.121, pp 31-42, 2018.

[21] T. M. Behera et al. "Hybrid heterogeneous routing scheme for improved network performance in WSNs for animal tracking," Internet of Things, Vol.6, 2019.

[22] H. Javed et al. "Grid-Based Hybrid Network Deployment Approach for Energy Efficient Wireless Sensor Networks," Journal of Sensors, Oct 2016.

[23] Abdul Waheed Khan, et al., "VGDRA: A Virtual Grid-Based Dynamic Routes Adjustment Scheme for Mobile Sink-Based Wireless Sensor Networks," IEEE Sensors Journal, Vol.15, No.1, pp 526 - 534, Jan 2015.

[24] Hossein Salmabadi, et al., "An improvement on LEACH protocol (EZ-LEACH)," 2nd International Conference on Knowledge-Based Engineering and Innovation (KBEI),_5-6 Nov 2015, Tehran, Iran.

[25] U. K. Paul and S. Chattopadhyay, "A novel grid based energy efficient routing algorithm for Wireless Sensor networks, " in International Conference on Wireless Communications, Signal Processing and Networking (WiSPNET), 23-25 March 2016, Chennai, India. 